\title{
Fisiopatología de la hipertensión asociada al síndrome de apnea obstructiva del sueño: Evidencia de estudios clínicos y modelos animales de hipoxia crónica intermitente
}

\author{
Sergio Rey ${ }^{1,2}$, G loria Valdés ${ }^{1}$, Rodrigo Iturriaga². \\ Pathophysiology of obstructive sleep \\ apnea-associated hypertension
}

There is a well established relationship between the obstructive sleep apnea syndrome and hypertension. Current evidence suggests that the increase in arterial pressure is secondary to an enhanced sympathetic tone through peripheral chemoreflexes triggered by intermittent hypoxic stimulation of the carotid bodies. Chronic intermittent hypoxia would activate renal and systemic vasoactive systems through potentiated hypoxic chemoreflexes. These early changes in autonomic tone can be detected through cardiovascular variability and baroreflex sensitivity analysis. Both are relatively simple and noninvasive techniques. The multiplicity of pathogenic mechanisms in obstructive sleep apnea-associated hypertension emphasizes the need of increasing diagnostic sensitivity to detect and correct this common condition, which significantly increases cardiovascular risk (Rev Méd Chile 2007; 135: 1333-42).

(Key w ords: Anoxemia; Hypertension; Sleep apnea, obstructive)

Recibido el 4 de septiembre, 2006. Aceptado el 23 de octubre, 2006.

Financiado por Fondecyt 1030330.

${ }_{1}^{1}$ Departamento de Nefrología, Facultad de Medicina. 2 Laboratorio de Neurobiología, Facultad de Ciencias Biológicas, Pontificia Universidad Católica de Chile, Santiago, Chile.

L a apnea obstructiva del sueño (AOS), síndrome caracterizado por episodios repetitivos de colapso de la vía aérea superior durante el sueño, afecta en promedio a $2 \%-4 \%$ de la población adulta $^{1,2}$. Se considera como apnea un período de interrupción de la respiración de más de diez

Correspondencia a: Dr. Sergio Rey. Departamento de Nefrología, Facultad de Medicina, Pontificia Universidad Católica de Chile. Lira 85, 4o piso, Santiago, Chile.

E-mail: srkeim@med.puc.cl segundos ${ }^{3}$. Los pacientes con AOS también pueden presentar hipopneas, que consisten en una disminución del flujo ventilatorio. El diagnóstico de AOS se hace cuando ocurren más de diez episodios de apnea, hipopnea o ambos por hora de sueño ${ }^{4}$, a pesar que $9 \%$ de las mujeres y $24 \%$ de los hombres presentan hasta cinco apneas por hora ${ }^{3,5}$. El colapso de la orofaringe con interrupción del flujo aéreo produce hipoxia e hipercapnia, estimulando los quimiorreceptores arteriales, que a su vez aumentan la presión intratorácica por activación de músculos respiratorios, tono vascular simpático, 
niveles de catecolaminas circulantes y presión arterial $^{6}$. Finalmente, la activación de quimio y mecanorreceptores produce un microdespertar (arousal), restaurando el flujo ventilatorio. Los pacientes con AOS severa pueden presentar más de 30 apneas por hora, llegando a repetirse unas 240 veces cada noche. La caída de la saturación de oxihemoglobina alcanza hasta $50 \%-60 \% 3$, que de acuerdo con la fórmula de Kelman ${ }^{7}$, equivalen a un rango de $\mathrm{PaO}_{2}$ entre $27-32 \mathrm{mmHg}$ a $37^{\circ} \mathrm{C}$. Los eventos apneicos producen fragmentación del sueño, causando somnolencia diurna ${ }^{8}$.

Varios estudios epidemiológicos han identificado a la AOS como un factor de riesgo independiente para el desarrollo de hipertensión arterial, infarto al miocardio, accidentes cerebrovasculares y muerte súbita ${ }^{5,9}$. El 50\%-90\% de los pacientes con AOS desarrollan hipertensión sistémica diurna ${ }^{10,11}$. Por otro lado, la AOS constituye una causa frecuente de hipertensión refractaria ${ }^{12}$. Estos pacientes presentan aumento del tono simpático ${ }^{13}$ e incremento de la descarga simpática periférica ${ }^{14}$. Además, los pacientes con AOS recién diagnosticada tienen una mayor reactividad vascular hipóxica ${ }^{15}$ y respuestas aumentadas de los quimiorreceptores periféricos a la hipoxia aguda ${ }^{16}$.

La asociación epidemiológica entre la AOS y obesidad, dificulta establecer una relación directa entre la AOS y la hipertensión. Sin embargo, se ha mostrado por análisis multivariado que existe una asociación independiente entre la AOS y la hipertensión ${ }^{17,18}$. En efecto, el Wisconsin Sleep Cohort Study ${ }^{19}$ mostró que existe una relación estadística independiente entre la severidad de la AOS y el desarrollo de hipertensión en cuatro años. Se sabe que la AOS causa elevaciones agudas de la presión arterial nocturna, que pueden alcanzar los 240/120 mmHg, y que son revertidas con presión positiva continua de la vía aérea (Continuous Positive Airway Pressure o CPAP) ${ }^{20}$. El tratamiento de la AOS logra disminuir tanto las presiones arteriales elevadas nocturnas como las diurnas, especialmente en individuos que presentan un mayor índice de severidad determinado por saturometría y que sufren somnolencia diuma ${ }^{21}$. En un estudio randomizado, doble ciego y controlado con placebo, este efecto fue más intenso tanto para presión sistólica, media y diastólica nocturna, como para diastólica y media diurna ${ }^{22}$. Consecuentemente, el último reporte de consenso del
Joint National Committee for Prevention, Detection, Evaluation and Treatment of High Blood Pressure $^{23}$, sugirió a la AOS como una de las primeras posibilidades a descartar como causa de hipertensión secundaria.

\section{MECANISMOS FISIOPATOLÓGICOS DE LA HIPERTENSIÓN ASOCIADA A AOS}

La evidencia clínica y experimental disponible sugiere que existen al menos tres componentes importantes en el desarrollo de la hipertensión asociada a AOS: 1) la potenciación de las respuestas reflejas hipóxicas por activación repetitiva de los quimiorreceptores del cuerpo carotídeo, 2) la hiperactividad simpática sostenida y 3) la disrregulación del balance de los sistemas vasoactivos que controlan la presión arterial.

\section{QUIMIORREFLEJOS ARTERIALES E HIPERACTIVIDAD SIMPÁTICA}

La regulación de la actividad simpática sobre el sistema cardiovascular depende de mecanismos humorales y neurogénicos, entre los que destacan los quimiorreceptores del cuerpo carotídeo y los barorreceptores del seno carotídeo. Motivados por la observación epidemiológica de la asociación entre AOS e hipertensión, Somers et al realizaron una serie de estudios que mostraron que pacientes con AOS recientemente diagnosticada, presentan activación tónica de los quimiorreflejos carotídeos ${ }^{15}$, mayor respuesta ventilatoria hipóxi$\mathrm{ca}^{16}$, descarga simpática periférica aumentada ${ }^{14}$ y alteraciones en la variabilidad cardiovascular ${ }^{24}$. Estos trabajos permitieron plantear que el aumento de la actividad simpática debido a la potenciación quimiosensorial, causaría el incremento de la presión arterial en los pacientes con AOS. Sin embargo, algunos estudios muestran resultados contradictorios sobre el papel de los quimiorreflejos en la hiperactividad simpática asociada a AOS, lo que podría deberse a la dificultad para separar y controlar factores como edad, tiempo de evolución de la AOS, comorbilidades, patrón hipóxico y tratamientos concomitantes 5 , que podrían producir variaciones en los resultados de los distintos estudios. Por lo tanto, para estudiar los mecanismos responsables de la generación de las respues- 
tas patológicas ventilatorias y cardiovasculares, se han usado modelos de exposición a hipoxia crónica intermitente (HCI) en animales. Los estudios realizados por Peng et $\mathrm{al}^{25,26}$, y por nosotros $6,27,28$, muestran que la exposición a HCI, por períodos cortos de 10 días en ratas y de 4 días en gatos, aumenta la descarga quimiosensorial basal y potencia las respuestas del cuerpo carotídeo a hipoxia aguda. Peng et $\mathrm{al}^{26}$ estudiaron los efectos de la HCI sobre la descarga quimiosensorial en ratas sometidas a episodios cíclicos de hipoxia $\left(4 \% \mathrm{O}_{2}\right.$ por $15 \mathrm{~s}$ seguidos por $5 \mathrm{~min}$ de normoxia, por 8 h durante 10 días). A los 10 días, la descarga basal y las respuestas quimiosensoriales producidas por hipoxia aumentaron, mientras que las respuestas a la hipercapnia fueron similares a los controles, indicando que la respuesta a la hipoxia -pero no a la hipercapnia- estaba aumentada por la HCI. Nosotros estudiamos las respuestas quimiosensoriales en gatos expuestos a episodios cíclicos de hipoxia de $10 \% \mathrm{O}_{2}$ por 2 min seguidos por 5 min de normoxia, por $8 \mathrm{~h}$ durante 4 días. Estudiamos, además, las respuestas quimiosensoriales inducidas por varios niveles de oxígeno ( $\mathrm{PO}_{2}$ entre 10-740 Torr). Los resultados muestran que la HCI mantenida por 4 días aumenta selectivamente las respuestas quimiosensoriales del cuerpo carotídeo de gato evocadas por la hipoxia aguda. Recientemente confirmamos estos resultados usando una preparación in vitro del cuerpo carotídeo, mostrando que la potenciación quimiosensorial es un fenómeno primario del cuerpo carotídeo, ya que el aumento de la reactividad se conserva después de extraerlo del animal ${ }^{28}$.

La rata se ha usado como modelo para estudiar los efectos de la HCI. Fletcher et $\mathrm{al}^{29}$ expusieron estos animales a ciclos de hipoxia/normoxia de un minuto de duración, con una $\mathrm{P}_{\mathrm{a}} \mathrm{O}_{2}$ mínima aproximada de $70 \%$ por $8 \mathrm{~h}$ al día durante 35 días. En estas condiciones, la presión arterial aumentó en $15 \mathrm{mmHg}$ y permaneció elevada durante varias semanas después de volver a normoxia. La hipertensión inducida por HCI fue abolida por la sección bilateral de los nervios carotídeos ${ }^{29}$ o por la denervación simpática renal ${ }^{30}$. Más recientemente, otros autores confirmaron que la HCI produce hipertensión, dependiente del patrón hipóxico y del tiempo de exposición ${ }^{31,32}$. Por lo tanto, la hipertensión inducida por HCI dependería de la estimulación simpática por los quimiorre- flejos carotídeos, y de la activación neurohumoral de sistemas vasoactivos renales, que regulan la presión arterial a lango plazo. La hiperactivación simpática observada tanto en modelos animales $^{33,34}$ como en pacientes con AOS 35 , contribuiría a una mayor morbimortalidad cardiovascular ${ }^{9}$, aumentando el riesgo de hipertensión arterial, falla cardíaca y accidentes vasculares encefálicos. En los casos más extremos, el aumento del tono simpático produce síntomas hiperadrenérgicos, hipertensión severa, elevación de las catecolaminas plasmáticas y normetanefrinas urinarias de tal magnitud que pueden simular feocromocitomas ${ }^{36}$. En estos pacientes, el tratamiento con CPAP revirtió los síntomas y la elevación de las catecolaminas 37 .

\section{CONTROL AUTONÓMICO DE LA PRESIÓN ARTERIAL Y VARIABILIDAD CARDIOVASCULAR EN LA AOS}

La frecuencia cardíaca depende de la descarga del nodo sinoatrial, el cual recibe inervación autonómica simpática y parasimpática ${ }^{38}$. La interacción de estas dos ramas del sistema nervioso autonómico sobre el corazón produce una variabilidad de la frecuencia cardíaca característica ${ }^{39}$. Lee y $\operatorname{Hon}^{40}$ fueron los primeros en describir que la alteración de la variabilidad del intervalo $\mathrm{RR}$ precede el sufrimiento fetal. Varios estudios posteriores describieron la existencia de ritmos fisiológicos contenidos en la señal de la frecuencia cardíaca ${ }^{41-43}$.

El análisis de la variabilidad de la frecuencia cardíaca detecta dos componentes oscilatorios de baja y alta frecuencia que se encuentran en fase con el tono vasomotor periférico y la respiración, respectivamente ${ }^{44,45}$. El componente de baja frecuencia (LF) se asocia con la actividad simpática vaso y cardiomotora ${ }^{46}$ y del sistema reninaangiotensina-aldosterona ${ }^{44}$. El componente de alta frecuencia (HF) está asociado a la modulación vagal de la frecuencia cardíaca y corresponde a la arritmia sinusal respiratoria ${ }^{45}$. En consecuencia, se ha propuesto que la razón $\mathrm{LF} / \mathrm{HF}$ sería un marcador del balance simpatovagal sobre la frecuencia cardíaca $^{45}$. Pagani et $\mathrm{al}^{47}$ mostraron una relación positiva entre el componente LF de la variabilidad de la frecuencia cardíaca y la descarga simpática neural hacia los músculos. En pacientes con AOS recién diagnosticada que no presentaban hiper- 
tensión, Narkiewicz et al $^{24}$ encontraron que la variabilidad total de la frecuencia cardíaca estaba disminuida, con un aumento significativo de la razón $\mathrm{LF} / \mathrm{HF}$. Estos pacientes también presentaban aumento de la descarga simpática periférica. De manera similar, Rey et $\mathrm{al}^{27}$ encontraron, en gatos, que la exposición a HCI por cuatro días potenciaba los quimiorreflejos carotídeos, las respuestas ventilatorias y la taquicardia inducida por la hipoxia aguda, aumentando significativamente la razón LF/ HF. Sin embargo, esta breve exposición no produjo hipertensión. En ratas, la exposición a la HCI por 30 días produce hipertensión, aumenta el componente LF de la variabilidad de la presión arterial y la razón $\mathrm{LF} / \mathrm{HF}^{32}$. Por lo tanto, es posible que la hiperactivación simpática sea un mecanismo relevante en la hipertensión arterial asociada a AOS.

Los baromeceptores arteriales regulan la actividad simpática y parasimpática sobre el sistema cardiovascular y contribuyen a la homeostasis cardiocirculatoria, principalmente en el corto plazo ${ }^{48}$. Varios estudios en pacientes con AOS han mostrado una disminución de la sensibilidad del baromeflejo ${ }^{49-51}$. Lai et $\mathrm{al}^{32}$ mostraron una disminución similar de la sensibilidad del baromeflejo en ratas expuestas a HCI.

Queremos enfatizar la relación entre hipoxia e hiperactividad simpática mostrando un registro de saturometría y frecuencia cardíaca en un paciente con AOS, y en una preparación experimental en el gato anestesiado. En el primer caso, destaca la relación inversa entre presión de $\mathrm{O}_{2}$ y frecuencia cardíaca, mientras que en el modelo animal se observa que la hipoxia aguda produce taquicardia e hipertensión arterial (Figura 1).

\section{MEDIADORES VASOACTIVOS E HIPERTENSIÓN ASOCIADA A} LA AOS

Otra hipótesis propuesta es que la HCI podría alterar el balance entre los sistemas vasoconstrictores y vasodilatadores que regulan la presión arterial, favoreciendo el desarrollo de hipertensión sostenida. La evidencia experimental ha mostrado cambios en al menos cuatro sistemas vasoactivos: endotelinas, renina-angiotensina-aldosterona, cali-
Figura 1. Efectos cardiovasculares de la HCI en humanos y modelos animales. A. Segmento de un registro de saturometría nocturna de hombre de 67 años con diagnóstico de AOS, en el que se observa una imagen en espejo de las curvas de $\mathrm{SpO}_{2}$ y frecuencia cardiaca $\left(f_{H}\right)$. B. Registro del efecto de distintos niveles de $\mathrm{O}_{2}$ inspirado (barras negras) sobre la frecuencia cardíaca y presión arterial $\left(\mathrm{P}_{\mathrm{a}}\right)$ en un gato macho anestesiado con pentobarbitona sódica. La reducción del $\mathrm{O}_{2}$ inspirado produce taquicardia e hipertensión. Línea gris, presión arterial media (Rey et al., datos no publicados).

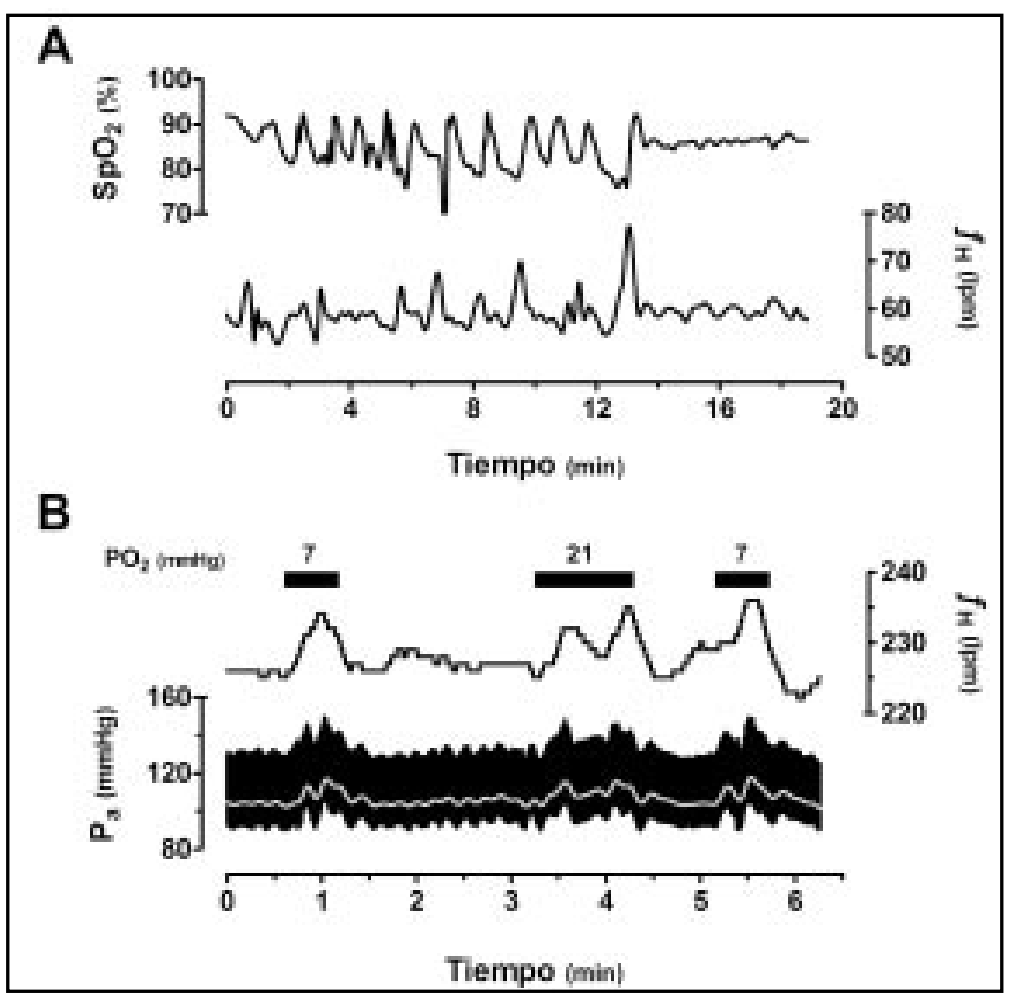


creína-cininas y óxido nítrico. Además, se ha reconocido un papel relevante del estrés oxidativo en la AOS.

Endotelinas. Dado que la ET-1 es uno de los vasoconstrictores más potentes que se conocen ${ }^{52}$, y que este péptido se libera por la estimulación hipóxica desde el endotelio vascular ${ }^{53}$, varios trabajos han explorado el papel de la ET-1 en la hipertensión arterial diurna que se observa en pacientes con AOS. Al menos dos estudios clínicos encontraron niveles elevados de ET-1 en el plasma de pacientes con $\mathrm{AOS}^{54,55}$, comparándolos con personas con sueño normal. El tratamiento con CPAP restauró los niveles plasmáticos normales de ET-1 en estos pacientes. En contraste, Grimpen et $\mathrm{al}^{56}$ no encontraron diferencias en los niveles plasmáticos de ET-1 al comparar pacientes con AOS con controles sanos corregidos por edad, sexo y peso, aunque no controlaron peso, severidad de la AOS y tratamientos recibidos, dificultando la interpretación de los datos.

El primer estudio enfocado al papel de la ET-1 en la hipertensión inducida por HCI fue realizado por Kanagy et $\mathrm{al}^{31}$. Estos investigadores encontraron que los niveles plasmáticos de ET-1 estaban elevados en ratas expuestas a HCI por 11 días, y que además este aumento se relaciona con hipertensión arterial, cuya aparición se pudo impedir mediante la administración de un bloqueador no selectivo de los receptores de endotelina. Sin embargo, es necesario hacer notar que la exposición a la hipoxia crónica sostenida también produce un aumento transitorio de los niveles de la ET-1 plasmática, aunque no induce hipertensión arterial ${ }^{57}$. Rey et $\mathrm{al}^{28}$ mostraron que la exposición de gatos a HCI por cuatro días inducía un aumento local de ET-1 inmunorreactiva en el cuerpo carotídeo, sin aumentar los niveles plasmáticos, sugiriendo que la vasoconstricción local en el cuerpo carotídeo produciría una disminución de la $\mathrm{PO}_{2}$ tisular en este quimiorreceptor, induciendo potenciación de los quimiorreflejos. Tomadas en conjunto, estas observaciones sugieren que hay variación en el curso temporal del aumento de la ET-1 plasmática de acuerdo al patrón de hipoxia crónica, y que probablemente existen otros mecanismos independientes de los niveles de ET-1 que contribuyen al desarrollo de hipertensión arterial en la HCI. Interesantemente,
Wong-Dusting et $\mathrm{al}^{58}$ encontraron que la ET-1 potenciaba la respuesta vasoconstrictora provocada por estimulación simpática y por noradrenalina en vasos arteriales. Fernández et $\mathrm{al}^{59}$ obtuvieron resultados similares en arterias de rata. Además, las endotelinas participarían en el control autonómico y en la homeostasis cardiovascular, actuando directamente sobre el sistema nervioso central ${ }^{60}$.

Sistema renina-angiotensina-aldosterona. Un estudio realizado en pacientes con AOS mostró mayores niveles de angiotensina II (AT-II) y de aldosterona comparados con pacientes sanos. Además, los autores encontraron que el tratamiento con CPAP redujo la actividad de renina plasmática y los niveles de AT-II ${ }^{61}$. Fletcher et $\mathrm{al}^{30}$ encontraron que la hipertensión inducida por HCI en ratas se acompaña de un aumento de la actividad de renina plasmática; además, la hipertensión no se desarrolló en ratas con denervación simpática renal o tratamiento con losartan, antagonista del receptor de angiotensina AT1. Recientemente, Yuan et al ${ }^{62}$ mostraron que la exposición a HCI por 42 días induce un aumento temprano en la actividad de renina plasmática (7 días) y luego, incrementa los niveles de AT-II (21 días), pero no modifica los niveles de mRNA del receptor AT1. Por lo tanto, uno de los mecanismos probables en la hipertensión asociada a AOS es una mayor actividad del sistema renina-angiotensina-aldosterona, principalmente a través de un aumento de los niveles de AT-II.

Sistema calicreína-cininas. Thongboonkerd et $\mathrm{al}^{63}$ caracterizaron la expresión proteica del sistema calicreína-cininas en un modelo de HCI en la rata. El análisis por proteómica mostró una disminución en la expresión de calistatina (un potente vasodilatador), pero con mantención de los niveles de calicreína y del receptor de bradicinina $B_{2}$, lo que favorecería una tendencia a la vasoconstricción. Dos trabajos han descrito una menor vasodilatación inducida por bradicinina en pacientes con AOS $^{64-65}$.

Oxido nítrico. Duchna et $\mathrm{al}^{64}$ encontraron que la vasodilatación mediada por el endotelio estaba reducida al comparar pacientes con AOS con individuos sanos. Sin embargo, no detectaron diferencias en la vasodilatación directa inducida 
por nitroglicerina. Se ha comprobado disminución del efecto vasodilatador de acetilcolina en el flujo del antebrazo, y niveles circulantes de óxido nítrico. Varios trabajos han reportado una disminución de los niveles de óxido nítrico e inhibición de la óxido nítrico sintasa en pacientes con $\mathrm{AOS}^{66-}$ 68. En la mayoría de los casos, el tratamiento con CPAP restauraba los niveles normales de óxido nítrico.

Estrés oxidativo. Se ha propuesto que los ciclos de hipoxia-reoxigenación que caracterizan la HCI son similares al fenómeno de isquemia-reperfusión, caracterizado por un aumento de la producción de especies reactivas del oxígeno durante la restitución del flujo sanguíneo. En los pacientes con AOS, el estrés oxidativo puede prolongarse por décadas, lo que hace posible que exista un daño oxidativo acumulativo que actuaría como un potente factor de riesgo cardiovascular. De acuerdo con esta hipótesis, Lavie et al, mostraron una correlación directa entre los niveles de dos marcadores de estrés oxidativo en el plasma (TBARS y peróxidos) y el índice apnea-hipopnea ${ }^{69}$, relación que se eliminó con el tratamiento con CPAP. Otro trabajo utilizó la excreción urinaria de 8-hidroxi2'-deoxiguanosina como medida de estrés oxidativo in vivo, mostrando una correlación positiva independiente entre los niveles de esta molécula con el índice apnea-hipopnea, mediante análisis multivariado ${ }^{70}$.

Entre otros factores que elevarían la presión arterial, se encuentra la resistencia a la insulina ${ }^{71,72}$ y los niveles plasmáticos de leptina ${ }^{73}$.

\section{CONCLUSIÓN Y PROYECCIONES}

La evidencia disponible sugiere que los ciclos de hipoxia-reoxigenación de la HCI producen una potenciación temprana de los quimiorreflejos, que contribuirían a aumentar la actividad simpática cardíaca y vasomotora. Los resultados obtenidos en modelos animales muestran que la HCI produce cambios tempranos en el balance autonómico sobre la frecuencia cardíaca y una atenuación de la sensibilidad de los barorreflejos. En forma similar, los pacientes con AOS presentan quimiorreflejos periféricos potenciados y una disminución de la sensibilidad de los barorreflejos. Por lo tanto, es posible que estas alteraciones favorezcan una mayor respuesta presora hipóxica y una mayor actividad simpática tónica, facilitando el desarrollo de hipertensión arterial. Además, la AOS se caracteriza por respuestas vasomotoras alteradas, con tendencia a la vasoconstricción. Las alteraciones fisiopatológicas en los sistemas de las endotelinas, renina-angiotensina-aldosterona, calicreína-cininas y en la síntesis de óxido nítrico facilitarían la vasoconstricción sistémica y el aumento de la presión arterial (Figura 2). Estos sistemas vasoactivos son posibles blancos terapéuticos para la reducción del riesgo cardiovascular en la AOS.

Proponemos que el análisis de la variabilidad cardiovascular y de la sensibilidad del barorreflejo son de utilidad en el diagnóstico inicial de los cambios que conducen a la hipertensión asociada a AOS, como métodos no invasivos y de bajo costo para detectar los cambios autonómicos asociados a la HCI.

La AOS debe plantearse como uno de los primeros factores a descartar en pacientes obesos con hipertensión refractaria, roncadores, con somnolencia diurna, en personas que no presentan descenso nocturno en los monitoreos ambulatorios de presión arterial y en aquellos pacientes sin patología pulmonar que presentan hematocrito elevado o en aumento a lo largo del tiempo. Deseamos enfatizar la necesidad de aumentar la sensibilidad clínica para sospechar el diagnóstico de AOS en sujetos hipertensos. 


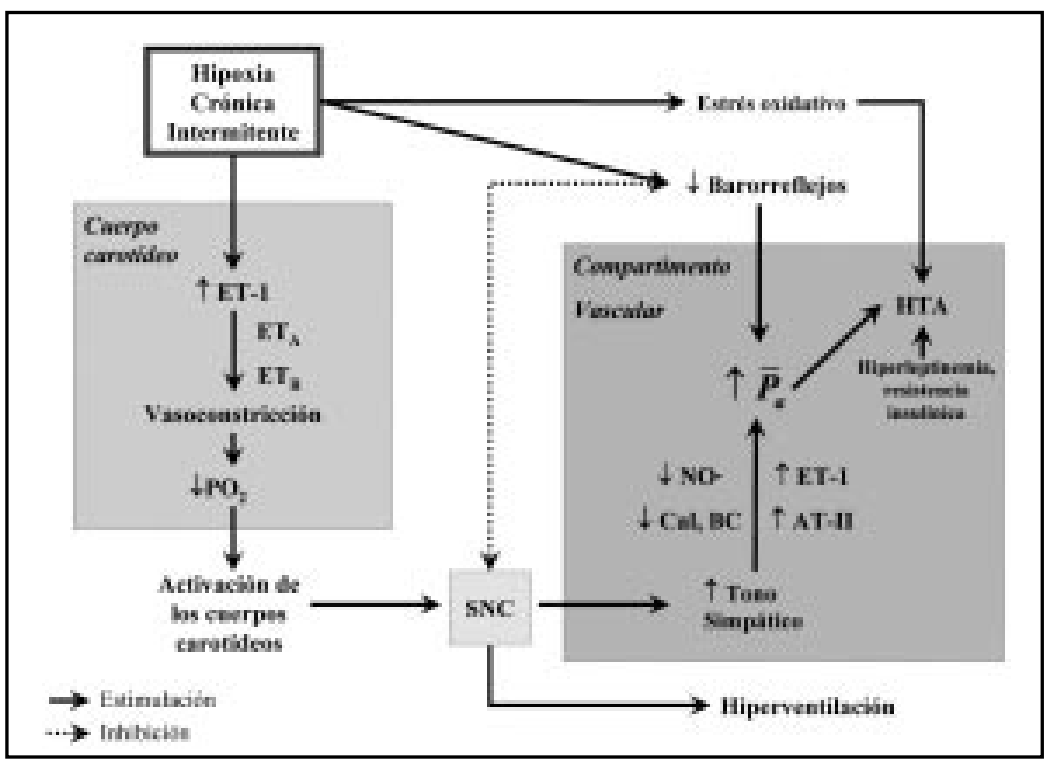

Figura 2. Mecanismos fisiopatológicos de la hipertensión asociada a la apnea obstructiva del sueño. La hipoxia crónica intermitente induce potenciación de los quimiorreflejos a través de vasoconstricción local en el cuerpo carotídeo, que produce caída de la presión parcial de oxígeno y aumenta la respuesta de los quimiorreceptores a la hipoxia. Los quimiorreflejos potenciados, contribuirían a una inhibición del barorreflejo. Estas alteraciones, al sostenerse en el tiempo inducirían un aumento de la presión arterial e hiperactividad simpática sostenidas. Además, estos cambios se acompañarían de aumento de la actividad de sistemas vasoconstrictores y disminución de los sistemas vasodilatadores. ET-1, endotelina-1; $\mathrm{ET}_{\mathrm{A} / \mathrm{B}}$, receptores de endotelina tipo A/B; $\mathrm{PO}_{2}$, presión parcial de oxígeno tisular; $\mathrm{SNC}$, sistema nervioso central; $\mathrm{P}_{\mathrm{a}}$ presión arterial media; $\mathrm{NO}$, óxido nítrico; Cal, calicreína; BC, bradicinina; AT-II, angiotensina II; HTA, hipertensión arterial.

\section{REFERENCIAS}

1. Partinem M. Epidemiology of obstructive sleep apnea syndrome. Curr Opin Pulm Med 1995; 1: 482-7.

2. Young T, Palta M, Dempsey J, Skatrud J, Weber $\mathrm{S}$, BADR S. The occurrence of sleep-disordered breathing among middle-aged adults. N Engl J Med 1993; 328: 1230-5.

3. Stroшo PJ, JR, Rogers RM. Obstructive sleep apnea. N Engl J Med 1996; 334: 99-104.

4. American Academy of SleEp Medicine. Sleep-related breathing disorders in adults: recommendations for syndrome definition and measurement techniques in clinical research. The Report of an American Academy of Sleep Medicine Task Force. Sleep 1999; 22: 667-89.

5. Hia KM, Young TB, Bidwell T, Palta M, Skatrud JB, DemPSEY J. Sleep apnea and hypertension. A population-based study. Ann Intern Med 1994; 120: $382-8$.

6. IturRiaga R, Rey S, Del Río R. Cardiovascular and ventilatory acclimatization induced by chronic intermittent hypoxia: a role for the carotid body in the pathophysiology of sleep apnea. Biol Res 2005; 38: 335-40.

7. KeLman GR. Digital computer subroutine for the conversion of oxygen tension into saturation. J Appl Physiol 1966; 21: 1375-6.

8. BRooks D, Horner RL, Kimoff RJ, Kozar LF, RenderTeixeira CL, Phiшipson EA. Effect of obstructive sleep apnea versus sleep fragmentation on responses to airway occlusion. Am J Respir Crit Care Med 1997; 155: 1609-17.

9. QuAN SF, GERSH BJ. Cardiovascular consequences of sleep-disordered breathing: past, present and future: report of a workshop from the National Center on Sleep Disorders Research and the 
National Heart, Lung, and Blood Institute. Circulation 2004; 109: 951-7.

10. Milman RP, Redune S, CarLsie CC, Assaf AR, LeVINSON PD. Daytime hypertension in obstructive sleep apnea. Prevalence and contributing risk factors. Chest 1991; 99: 861-6.

11. Roux F, D’Ambrosio C, Mohsenin V. Sleep-related breathing disorders and cardiovascular disease. Am J Med 2000; 108: 396-402.

12. Logan AG, Tkacova R, PerdKowski SM, Leung RS, Tisler A, FLORAS JS, BRADLEY TD. Refractory hypertension and sleep apnoea: effect of CPAP on blood pressure and baroreflex. Eur Respir J 2003; 21: 241-7.

13. SMith ML, Muenter NK. Effects of hypoxia on sympathetic neural control in humans. Respir Physiol 2000; 121: 163-71.

14. Narkiewicz K, Van de Borne PJ, Cooley RL, Dyken ME, SOMERS VK. Sympathetic activity in obese subjects with and without obstructive sleep apnea. Circulation 1998; 98: 772-6.

15. Narkiewicz K, Van de Borne PJ, Montano N, Dyken ME, PhiшPs BG, Somers VK. Contribution of tonic chemoreflex activation to sympathetic activity and blood pressure in patients with obstructive sleep apnea. Circulation 1998; 97: 943-5.

16. Narkiewicz K, Van de Borne PJ, Pesek CA, Dyken ME, MONTANo N, SOMERS VK. Selective potentiation of peripheral chemoreflex sensitivity in obstructive sleep apnea. Circulation 1999; 99: 1183-9.

17. Robinson GV, Strading JR, Davies RJ. Obstructive sleep apnoea/hypopnoea syndrome and hypertension. Thorax 2004; 59: 1089-94.

18. Wolk R, Shamsuzzaman AS, Somers VK. Obesity, sleep apnea, and hypertension. Hypertension 2003; 42: 1067-4.

19. Peppard PE, Young T, Palta M, Skatrud J. Prospective study of the association between sleepdisordered breathing and hypertension. N Engl J Med 2000; 342: 1378-84.

20. SOMERs VK, Dyken ME, Clary MP, Abboud FM. Sympathetic neural mechanisms in obstructive sleep apnea. J Clin Invest 1995; 96: 1897-904.

21. Pepperell JC, Ramdassingh-Dow S, Crosthwaite N, Mumins R, Jenkinson C, Strading JR, Davies RJ. Ambulatory blood pressure after therapeutic and subtherapeutic nasal continuous positive airway pressure for obstructive sleep apnoea: a randomized parallel trial. Lancet 2002; 359: 204-10.

22. Norman D, Loredo JS, Nelesen RA, Ancol-Israel S, Mius PJ, Ziegler MG, Dimsdale JE. Effects of continuous positive airway pressure versus supplemental oxygen on 24-hour ambulatory blood pressure. Hypertension 2006; 47: 840-5.

23. Chobanian AV, Bakris GL, Biack HR, Cushman WC, Green LA, Izzo JL JR ET aL. The Seventh Report of the Joint National Committee on Prevention, Detection, Evaluation, and Treatment of High Blood Pressure: the JNC 7 report. JAMA 2003; 289: 2560-72.

24. Narkiewicz K, Montano N, Coglati C, Van de Borne PJ, Dyken ME, Somers VK. Altered cardiovascular variability in obstructive sleep apnea. Circulation 1998; 98: 1071-7.

25. Peng YJ, Prabhakar NR. Reactive oxygen species in the plasticity of respiratory behavior elicited by chronic intermittent hypoxia. J Appl Physiol 2003; 94: 2342-9.

26. Peng YJ, Prabhakar NR. Effect of two paradigms of chronic intermittent hypoxia on carotid body sensory activity. J Appl Physiol 2004; 96: 1236-42.

27. Rey S, Del Río R, Alcayaga J, IturRiaga R. Chronic intermittent hypoxia enhances cat chemosensory and ventilatory responses to hypoxia. J Physiol 2004; 560: 577-86.

28. Rey S, Del Río R, Iturriaga R. Contribution of endothelin-1 to the enhanced carotid body chemosensory responses induced by chronic intermittent hypoxia. Brain Res 2006; 1086: 152-9.

29. Fietcher EC, Lesske J, BeHm R, Miuer CC, $3^{\text {RD }}$, Stauss H, UNGER T. Carotid chemoreceptors, systemic blood pressure, and chronic episodic hypoxia mimicking sleep apnea. J Appl Physiol 1992; 72: 1978-84.

30. FIETCHeR EC BAO G, LI R. Renin activity and blood pressure in response to chronic episodic hypoxia. Hypertension 1999; 34: 309-14.

31. Kanagy NL, Walker BR, Nelin LD. Role of endothelin in intermittent hypoxia-induced hypertension. Hypertension 2001; 37: 511-5.

32. Lai CJ, Yang CC, Hsu YY, Ln YN, Kuo TB. Enhanced sympathetic outflow and decreased baroreflex sensitivity are associated with intermittent hypoxia-induced systemic hypertension in conscious rats. J Appl Physiol 2006; 100: 1974-82.

33. FLETCHER EC. Effect of episodic hypoxia on sympathetic activity and blood pressure. Respir Physiol 2000; 119: 189-197.

34. Greenberg HE, Sica A, Batson D, Scharf SM. Chronic intermittent hypoxia increases sympathetic responsiveness to hypoxia and hypercapnia. J Appl Physiol 1999; 86: 298-305. 
35. LeUenberger UA, Brubaker D, Quraichi S, Hogeman CS, ImADOJEMU VA, Gray KS. Effects of intermittent hypoxia on sympathetic activity and blood pressure in humans. Auton Neurosci 2005; 121: 87-93.

36. Hoy LJ, Emery M, Wedzocha JA, Davison AG, Chew SL, Monson JP, Metcalfe KA. Obstructive sleep apnea presenting as pseudopheochromocytoma: a case report. J Clin Endocrinol Metab 2004; 89: 2033-8.

37. Makino S, Imata M, Fujiwara M, Ike S, Tateyama H. A case of sleep apnea syndrome manifesting severe hypertension with high plasma norepinephrine levels. Endocr J 2006; 53: 363-9.

38. LeVY MN, Martin PJ, Stuesse SL. Neural regulation of the heart beat. Annu Rev Physiol 1981; 43: 44353.

39. Kamath MV, FaLien EL. Power spectral analysis of heart rate variability: a noninvasive signature of cardiac autonomic function. Crit Rev Biomed Eng 1993; 21: 245-311.

40. LeE ST, Hon EH. Electronic evaluations of the fetal heart rate preceeding fetal death, further observations. Am J Obstet Gynecol 1965; 87: 814-26.

41. HiRsCh JA, Bishop B. Respiratory sinus armythmia in humans: how breathing pattern modulates heart rate. Am J Physiol 1981; 241: H620-9.

42. LuCZAK H, LAURIG W. An analysis of heart rate variability. Ergonomics 1973; 16: 85-97.

43. SAYERS BM. Analysis of heart rate variability. Ergonomics 1973; 16: 17-32.

44. Akselrod S, Gordon D, Ubel FA, Shannon DC, BERGER AC, CoHEN RJ. Power spectrum analysis of heart rate fluctuation: a quantitative probe of beat-to-beat cardiovascular control. Science 1981; 213: 220-2.

45. Task Foorce of the European Society of Cardiology and the North American Society of Pacing and EleCtro phy Siology. Heart rate variability. Standards of measurement, physiological interpretation, and clinical use. Eur Heart J 1996; 17: 354-81.

46. Pomeranz B, Macaulay RJ, Caudill MA, Kutz I, Adam D, GoRdon D ET aL. Assessment of autonomic function in humans by heart rate spectral analysis. Am J Physiol 1985; 248: H151-3.

47. Pagani M, Montano N, Porta A, Mamini A, Abboud FM, BIRKETT C, SOMERS VK. Relationship between spectral components of cardiovascular variabilities and direct measures of muscle sympathetic nerve activity in humans. Circulation 1997; 95: 1441-8.
48. THRASHER TN. Baroreceptors and the long-term control of blood pressure. Exp Physiol 2004; 89: 331-5.

49. Bonsignore Mr, Parati G, Insalaco G, Marrone O, Castigloni P, Romano S et al. Continuous positive airway pressure treatment improves baroreflex control of heart rate during sleep in severe obstructive sleep apnea syndrome. Am J Respir Crit Care Med 2002; 166: 279-286.

50. Jo Ja, Biasi A, Valladares E, Juárez R, Baydur A, Kноо MC. Determinants of heart rate variability in obstructive sleep apnea syndrome during wakefulness and sleep. Am J Physiol Heart Circ Physiol 2005; 288: H1103-12.

51. Parato G, Di Rienzo M, Bonsignore MR, Insalaco G, Marrone O, Castigloni P et al. Autonomic cardiac regulation in obstructive sleep apnea syndrome: evidence from spontaneous baroreflex analysis during sleep. J Hypertens 1997; 15: 1621-6.

52. DaVENPORT AP. International Union of Pharmacology. XXIX. Update on endothelin receptor nomenclature. Pharmacol Rev 2002; 54: 219-26.

53. Elton TS, Oparil S, Taylor GR, Hicks PH, Yang RH, JiN H, CHEN YF. Normobaric hypoxia stimulates endothelin-1 gene expression in the rat. Am J Physiol 1992; 263: R1260-4.

54. Phimps BG, Narkiewicz K, Pesek CA, Haynes WG, DYKEN ME, SOMERS VK. Effects of obstructive sleep apnea on endothelin-1 and blood pressure. J Hypertens 1999; 17: 61-6.

55. Saarelainen S, Seppala E, Laasonen K, Hasan J. Circulating endothelin-1 in obstructive sleep apnea. Endothelium 1997; 5: 115-8.

56. Grimpen F, Kanne P, Schuiz E, Hagenah G, Hasenfuss G, Andreas S. Endothelin-1 plasma levels are not elevated in patients with obstructive sleep apnoea. Eur Respir J 2000; 15: 320-5.

57. Ni Z, Bemanian S, KivLighn SD, Vaziri ND. Role of endothelin and nitric oxide imbalance in the pathogenesis of hypoxia-induced arterial hypertension. Kidney Int 1998; 54: 188-92.

58. Wong-Dusting HK, La M, Rand MJ. Endothelin-1 enhances vasoconstrictor responses to sympathetic nerve stimulation and noradrenaline in the rabbit ear artery. Clin Exp Pharmacol Physiol 1991; 18: 131-6.

59. Fernández N, Sánz E, Monge L, Martínez MA, Dieguez G, García-Vilialón AL. Potentiation by endothelin-1 and vasopressin of sympathetic vasoconstriction in male and female rats. Clin Sci (Lond) 2002; 103: 158S-161S. 
60. KuwaKi T, KuRIHARa H, CaO WH, UneKaWA M, YazaKI Y, KuMADA M. Physiological role of brain endothelin in the central autonomic control: from neuron to knockout mouse. Prog Neurobiol 1997; 51: 545-79.

61. Moler DS, Lind P, Strunge B, Pedersen EB. Abnormal vasoactive hormones and 24-hour blood pressure in obstructive sleep apnea. Am J Hypertens 2003; 16: 274-80.

62. Yuan ZM, Chen BY, WANG PX, Li SY, Chen YL, DoNG LX. Changes of angiotensin II and its receptor during the development of chronic intermittent hypoxia-induced hypertension in rats. Zhonghua Jie He He Hu Xi Za Zhi 2004; 27: 577-80.

63. Thongboonkerd V, Gozal E, Sachleben LR, JR, arthur JM, Pierce WM, Cai J et al. Proteomic analysis reveals alterations in the renal kallikrein pathway during hypoxia-induced hypertension. J Biol Chem 2002; 277: 34708-16.

64. Duchna HW, Orth M, Schultze-Werninghaus G, GuiLe C, Sтоонs RA. Long-term effects of nasal continuous positive airway pressure on vasodilatory endothelial function in obstructive sleep apnea syndrome. Sleep Breath 2005; 9: 97-103.

65. Duchna HW, Orth M, Schultze-Werninghaus G, Guileminault C, Sтоонs RA. Antihypertensive treatment and endothelium-dependent venodilation in sleep-disordered breathing. Sleep Breath 2006; 10:115-22.

66. Ip MS, Lam B, Chan LY, Zheng L, Tsang KW, Fung PC, LAM WK. Circulating nitric oxide is suppres- sed in obstructive sleep apnea and is reversed by nasal continuous positive airway pressure. Am J Respir Crit Care Med 2000; 162: 2166-71.

67. OHiKe Y, Kozaki K, IJjma K, Ето M, Kojima T, OHga E ET AL. Amelioration of vascular endothelial dysfunction in obstructive sleep apnea syndrome by nasal continuous positive airway pressure-possible involvement of nitric oxide and asymmetric NG, NGdimethylarginine. Circ J 2005; 69: 221-6.

68. Teramoto S, Kume H, Matsuse T, Ishi T, Miyashita A, AKISHITA M ET AL. Oxygen administration improves the serum level of nitric oxide metabolites in patients with obstructive sleep apnea syndrome. Sleep Med 2003; 4: 403-7.

69. Lavie L, Vishnevsky A, Lavie P. Evidence for lipid peroxidation in obstructive sleep apnea. Sleep 2004; 27: 123-8.

70. Yaumauchi M, Nakano $\mathrm{H}$, Maekawa J, Okamoto $\mathrm{Y}$, OHNISHI Y, SuZUKI T, KImURA H. Oxidative stress in obstructive sleep apnea. Chest 2005; 127: 1674-9.

71. Ip MS, Lam B, Ng MM, Lam WK, Tsang KW, Lam KS. Obstructive sleep apnea is independently associated with insulin resistance. Am J Respir Crit Care Med 2002; 165: 670-6.

72. Punjabi NM, Sorkin JD, Katzel LI, Goldberg AP, SchwarTZ AR, Smith PL Sleep-disordered breathing and insulin resistance in middle-aged and overweight men. Am J Respir Crit Care Med 2002; 165: 677-82.

73. Shek EW, Brands MW, Hall JE. Chronic leptin infusion increases arterial pressure. Hypertension 1998; 31: 409-14. 